\title{
DE LA LÓGICA A LA ÉTICA. LA SINFONÍA DEL LÓGOS EN ARISTÓTELES
}

\author{
Cécile Furstenberg*
}

Aristóteles alude a diversos escritos o trabajos en su tratado de Interpretación que forma parte del Organon o Tratado de Lógica: a la Retórica, a la Poética, a De Anima, pero no se encuentra ninguna referencia explícita a la Ética. Sin embargo la lectura en particular del capítulo 9 de de Interpretación sugiere una relación posible con la Ética. En efecto Aristóteles después de haber definido lo que es la oración

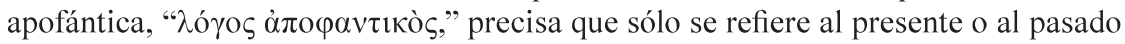
ya que para el futuro no se da la verdad o falsedad, la contingencia no obliga a la necesidad. Para Aristóteles las acciones humanas no son determinadas. En la Ética a Nicómaco, en particular en el capítulo 6, Aristóteles va a precisar lo que es la acción humana y cómo interviene la lógica en el proceso de deliberación, decisión, acción. Luego, resulta interesante precisar las relaciones entre la Lógica y la Ética en el conjunto de estas obras, lo que es correlativo con las relaciones entre la verdad y el bien, la teoría y la práctica. Se bosqueja un tema que fue rescatado en escritos de filósofos contemporáneos.

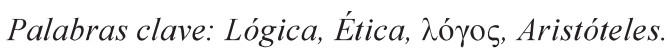

\section{FROM LOGIC TO ETHIC. SYMPHONY OF LOGOS IN ARISTOTLE}

\begin{abstract}
Aristotle alludes to diverse writings or works in his agreement on The Interpretation that does part of the Organon or agreement of Logic: to the Rhetoric, to the Poetics, to of Soul, but one does not find any explicit reference to the Ethics. Nevertheless the reading especially of the chapter 9 of The Interpretation Aristotle suggests a possible link with the Ethics in effect after having defined what is the apofantic sentence

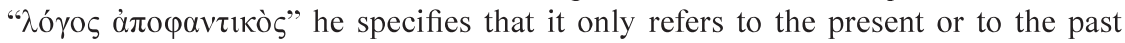
since for the future one does not give the truth or falsehood: the contingency does not force to the need. For Aristotle the human actions are not certain. In the Ethics to Nicómaco especially in the chapter 6 Aristotle is going to specify what is the human practice and as the logic intervenes in the process of deliberation, decision, action. Then it turns out interesting to note the relations between the Logic and the Ethics in the set of these works, which is correlative with the relations between the truth and the good, the theory and the practice. There is outlined a topic that was rescued in writings of contemporary philosophers.
\end{abstract}

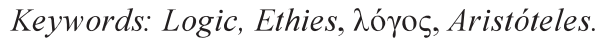

* Pontificia Universidad Católica de Chile 

De la Lógica a la Ética. La sinfonía del Lógos en Aristóteles

\section{Introducción}

ARISTÓteles, CON SU INTELIGENCIA, NO DEJABA ESCAPAR TEMAs fuera del alcance de sus estudios e intereses, polímata: lógico, científico, filósofo sus trabajos fomentaron comentarios, estudios, interés durante milenios, con interpretaciones variadas en los distintos continentes que no logran agotar la motivación para las investigaciones actuales. Filósofo de la antigüedad (384-322 a.C.), discípulo de Platón durante aproximadamente 20 años, activo en la Academia, se separó luego de su maestro, tomó distancia con la teoría de las Ideas de Platón y formó su propia escuela el Liceo. Andrónico de Rodas, hacia el año 60 a.C. hizo una recopilación de sus obras. El orden de clasificación escogido: el tratado de la Lógica, luego el de la Física, Metafísica, Ética, Política y por fin la Poética y la Retórica responde a una cierta coherencia que corresponde a la recepción y comprensión de los escritos en esta época. Sin embargo la autenticidad misma de algunos tratados fue puesta a prueba así como la cronología exacta, la certeza de la correspondencia con los escritos propios de Aristóteles es vaga, las modulaciones de los comentarios tempranos hacen surgir dudas, sin embargo se confirma una relación cierta con sus trabajos. Se vislumbra en cada tratado una peculiar atención de Aristóteles a crear vínculos y a hacer pertinentes referencias a otros tratados o temas de trabajo. En esta época los nexos entre temas y tratados de Física, Lógica e Ética, como partes de la filosofía o del aprendizaje filosófico para los estoïcos, se expresaban en forma metafórica. Así por ejemplo para Posidonio ${ }^{1}$ la filosofía es un cuerpo humano del cual la física es la sangre y la carne, la lógica: los huesos y tendones, y la ética: su alma.

El tratado aristotélico el "Organon", que corresponde a la denominación actual de Lógica es un "instrumento de trabajo", ofrece una metodología de reflexión. Comprende los libros siguientes: Las Categorías, de la Interpretación, los primeros analíticos, los analíticos posteriores, Tópico, de las Refutaciones Sofisticas. Desde los términos significantes se llega a las proposiciones y luego a las argumentaciones. Después de las Categorías que son "un point de rencontre de la chose dans sa manifestation et de la parole dans sa

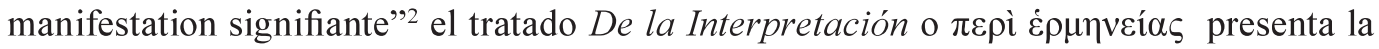

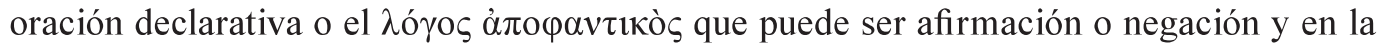

1 Boeri, M.- Salles, R., 2014. Los filósofos estoïcos. Ontología, lógica, física y ética. Universidad Alberto Hurtado.

2 Aubenque, P. (1980). « Préface ». En P. Aubenque (coord). Concepts et catégories dans la pensée antique. Paris: Vrin, $\mathrm{p}$ VIII. 
cual se da la verdad o la falsedad por medio del juicio. La denominación del tratado es posterior a Aristóteles. No se encuentra ninguna referencia a la palabra $\varepsilon \rho \mu \eta v \varepsilon i ́ \alpha$ en dicho tratado aunque para Aubenque ${ }^{3}$ se puede justificar la atribución de este título relacionado con la hermenéutica como correspondiendo al propósito de Aristóteles: la proposición declarativa es la interpretación del pensamiento. Efectivamente el titulo corresponde bien al capítulo primero donde se dice que las voces $\varphi$ ovñ y su traducción escrita expresan o traducen las representaciones $\pi \alpha \theta \eta \dot{\eta} \mu \alpha \tau \alpha \tau \tilde{\eta} \varsigma \psi v \chi \tilde{\eta} \varsigma$ de las cosas ( $\pi \rho \alpha ́ \gamma \mu \alpha \tau \alpha)$. En este tratado Aristóteles alude a temas abordados en la retórica, la poética o de Anima, no hace referencia alguna a la ética. Sin embargo se desprende del capítulo 9 en particular una relación interesante plausible que merece ser recalcada. Se presenta así, a Aristóteles, una aporía, ya que su lógica binaria, la cual otorga a toda oración declarativa de ser verdadera o falsa, sólo se puede confirmar para declaraciones relativas al pasado y al presente pero no se puede asegurar para las referidas al futuro. Aristóteles rechaza la determinación en las acciones humanas: "de modo que no fuera necesario decidir ( $\beta$ ov $\lambda \varepsilon v ́ \varepsilon \sigma \theta \alpha 1)$ algo o preocuparse por algo $(\pi \rho \alpha \gamma \mu \alpha \tau \varepsilon v ́ \varepsilon \sigma \theta \alpha \mathrm{l})$ (18b30). La contingencia no es necesaria por lo que está relacionado con las acciones humanas y depende de la decisión humana.

"Pues vemos que hay un principio de las cosas que serán y se encuentran en la decisión

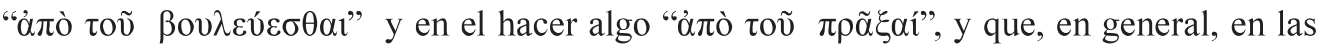
cosas que no siempre son de hecho existe lo posible y lo no posible, en la que ambas cosas pueden ocurrir: que sean o no sean, de modo que también el llegar a ser y el no llegar a ser" (19a8).

La relación entre la decisión y la praxis es en efecto el objeto de la ética y en particular del capítulo 6 de la Ética a Nicómaco. Luego se esboza una lectura enriquecida posible con los mutuos aportes de ambos tratados el de Interpretación y el de la ética con unas temáticas paralelas que se entrecruzan o entretejen: La lógica/ La ética, la verdad/ el bien, teoría/ practica, el lenguaje/ el deseo/ El juicio/la virtud. Quizás el hilo conductor pueda

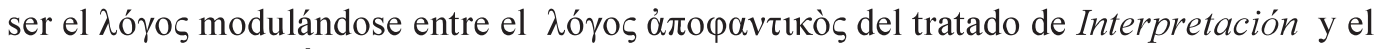
ó $\theta$ ós $\lambda o ́ \gamma o \varsigma$ de la Ética a Nicómaco.

En primer lugar presentaré más detalladamente la relación entre la Verdad y la proposición declarativa y el juicio, en de Interpretación. En segundo lugar expondré la orientación al Bien en la Ética, la función del deseo y la acción por el medio de la virtud, en $L a$ Ética a Nicómaco. En tercer lugar mostraré como las nociones no pueden ser consideradas separadamente y señalaré algunas conexiones interesantes para descubrir la sinfonía de la lógica y de la ética, de la verdad y del bien, de la teoría y de la práctica, sinfonía del logos. Identificaré también la notable incidencia de la relación aristotélica entre la lógica y la ética, la verdad y el bien en autores contemporáneos. Como conclusión, propondré unas perspectivas interesantes en el campo de la salud.

3 Aubenque, P. (2009). « Sens et unité du traité Aristotélicien de l'Interprétation». En Interpréter de Interpretatione de Suzanne Husson. Paris : Vrin, 37. 


\section{1) La Lógica y la verdad en de Interpretación. Función del lenguaje y del juicio. El

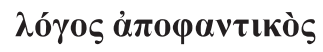

En el tratado de las Categorías $^{4}$ se inaugura lo que va a ser presentado como más detalladamente como " $\lambda o ́ \gamma o \varsigma \alpha$ à de describir las diez categorías, Aristóteles precisa que de los ítems citados no se da la

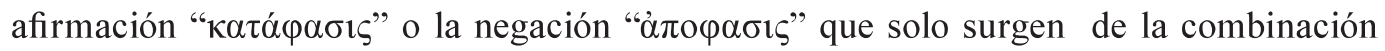

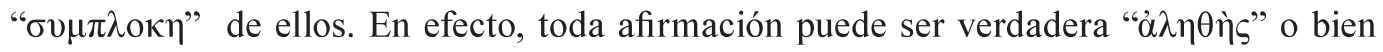

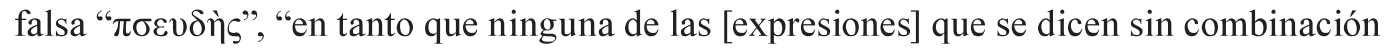
es verdadera o falsa, como por ejemplo "hombre", "blanco", "corre", "vence" (2a10)". Se nota la importancia de la combinación que será presentada en forma más precisa en el tratado de Interpretación como la unión de un nombre "sonido vocal que significa por

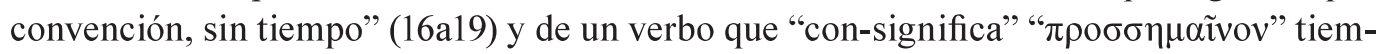
po (16b6), según la traducción acertada de Ammonio". El " $\lambda o ́ \gamma o \varsigma$ " es un sonido vocal

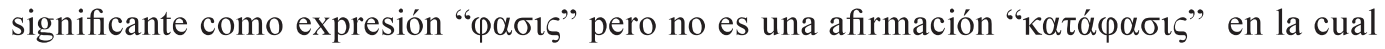
se da la verdad y la falsedad.

En las Categorías Aristóteles también se refirió a la afirmación y a la negación en los predicamentos cuando aborda las oposiciones. Cuando presenta la posesión y la privación como un tipo de oposiciones dice que la oposición es similar a la de la afirmación y de la negación. "Se dice, sin embargo, que también tales cosas se oponen entre sí como la afirmación y la negación, dado que el tipo de oposición es el mismo" (12b6). Luego, Aristóteles explica lo que son los contrarios y explica que si hay combinación, por ejemplo, Sócrates es sano y su contrario Sócrates está enfermo, uno será verdadero y lo otro falso, pero solamente si Sócrates existe. "De tal manera que solamente será propio de cuantas cosas se oponen entre sí al modo de la afirmación y la negación el que siempre una de las dos sea verdadera o falsa" (13b35).

Siguiendo la lectura de las Categorías llegamos a la parte dedicada a la anterioridad y sus modos de expresión. La anterioridad es también expresión de causalidad, por ejemplo en las ciencias. Es interesante aclarar que Aristóteles clasifica la cosa como anterior al enunciado de cuya verdad o falsedad no dependerá la existencia o no de la cosa. La anterioridad es también expresión de causalidad. "Sin embargo, el enunciado verdadero

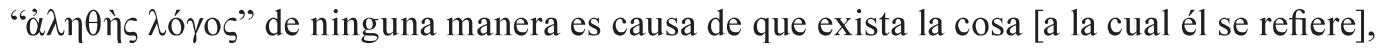
mientras que la cosa parece ser en cierto modo causa de que el enunciado sea verdadero, puesto que, por existir o no la cosa, el enunciado se dice verdadero o falso" (14b20). Aristóteles define la verdad como adecuación con la cosa " $\pi \rho \tilde{\alpha} \gamma \mu \alpha$ ". En distintos escritos DI, 18a39-b3 y en Metafísica, IX.10.1051b5-9 se encuentra esta definición de verdad que, sin

4 Referencias de la traducción en español de Mittelmann, J. (2009). Aristóteles. Las Categorías y sobre la Interpretación. Buenos Aires: Losadas. Referencias en griego de Cooke. (1983).

5 Joly, A. (2008). « Notes de lectures: de quelques malentendus sur la définition du verbe ». ERIS : 141 
embargo no es su única forma de expresión, ya que veremos que en la Ética a Nicómaco la verdad es más bien ontológica. El significado de "cosa" " $\pi \rho \tilde{\alpha} \gamma \mu \alpha$ " en la antigüedad ha evolucionado, Hadot ${ }^{6}$ subraya el interés de compilar los distintos significados ya que la connotación de materia asociada a la "cosa" en el lenguaje actual no era tan evidente en esta época. La traducción como "cosa" es vaga y puede prestar a confusión. Aristóteles diferencia el " $\lambda o ́ \gamma o \zeta "$ del " $\pi \rho \tilde{\alpha} \gamma \mu \alpha$ " en Las Categorias $(12 b 14,14 b 21)$, el " $\lambda o ́ \gamma o \varsigma$ " es la frase como dicción y el " $\pi \rho \tilde{\alpha} \gamma \mu \alpha$ " es "de lo que se habla" o "el asunto del cual se trata". En de Interpretación se encuentra "En cuanto a las" $\pi \rho \tilde{\alpha} \gamma \mu \alpha \tau \alpha$ " los unos son universales, los otros particulares (17a38)"o se refiere a "las cosas" como sujetos: "vं (21b28). Si Aristóteles da una connotación más bien concreta de las cosas, no le asigna una definición estrictamente material.

En de Interpretación ${ }^{7}$ Aristóteles explica más sutilmente como se da la verdad o falsedad. Introduce luego que hay "vó $\mu \mu \alpha$ " en nuestra mente, que no son relacionados con la verdad y falsedad y otros lo son. Confirma la necesidad de la adición de un verbo, que con-significa el tiempo, al nombre para que por la combinación " $\sigma v ́ v \theta \varepsilon \sigma ı \iota$ " y separación

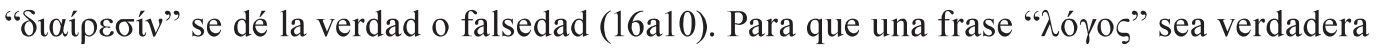
o falsa tiene que ser una proposición compuesta por un nombre y un verbo. El verbo ser por ejemplo que añade la existencia al sujeto predicado permite la calificación de " $\lambda$ ó $\gamma o \varsigma$ $\dot{\alpha} \pi \circ \varphi \alpha \nu \tau 1 \kappa o ̀ \varsigma "$ en que se expresa la verdad y la falsedad. "En efecto, toda proposición

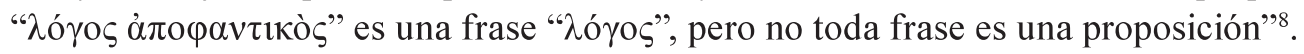

\section{¿Cuál es el paso de "la cosa" a la palabra, al " $\lambda o ́ \gamma o \varsigma \alpha ̉ \pi o \varphi \alpha v \tau$}

El recorrido, de "las cosas" " $\pi \rho \alpha ́ \gamma \mu \alpha \tau \alpha$ " a las representaciones en la mente " $\pi \alpha \theta \eta \dot{\eta} \mu \alpha \tau \alpha$

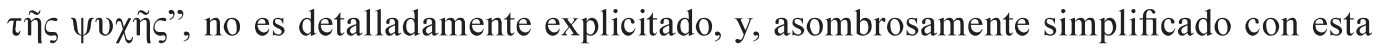
expresión que es "igual para todos". Lo importante es recalcar que estas representaciones son semejantes, "ó $\mu$ ot $\mu \alpha \tau a$ ", a las cosas. Aubenque 9 determina dos pasos: uno de las cosas a las representaciones en la mente, por semejanza; el otro de las representaciones a las palabras como símbolos de dichas representaciones, por traducción. La síntesis o verdad antepredicativa es distinta de la verdad obtenida en la oración declarativa en que hay posibilidad de contradicción. Esta verdad antepredicativa se funda según Moreau ${ }^{10}$ en la intencionalidad del pensamiento.

6 Надот, P. (1980) « Sur divers sens du mot pragmata dans la tradition philosophique grecque ». En Concepts et catégories dans la pensée Antique ». Por Pierre Aubenque, Paris: Vrin, p312.

7 Referencias en español Correia, M. (2003). La lógica de Aristóteles. Santiago: Ediciones Universitarias Católica de Chile: Apéndice 2. Referencias del texto griego de Weidemann, H.. (2014). Aristóteles de Interpretatione. Berlin: De Gruyter.

8 Correia, M. (2003). La lógica de Aristóteles. Santiago: Ediciones Universitarias Católica de Chile, p. 59.

9 Aubenque, P. (2009). «Sens et unité du traité Aristotélicien de l'Interprétation». En Interpréter de Interpretatione de Suzanne Husson. Paris : Vrin, pp. 37-50.

10 Moreau, J. (1980). " Aristote et la vérité antéprédicative ». En Aristote et les problèmes de méthodes. Paris: Vrin, pp. 21-33. 
El paso de las representaciones en el alma a la palabra se realiza por traducción y según una convención " $\kappa \alpha \tau \alpha \sigma v v \theta \eta ́ \kappa \eta "$ ". Gracias a la combinación del nombre y del verbo se obtiene la oración declarativa y por el medio de la composición y separación se da la false-

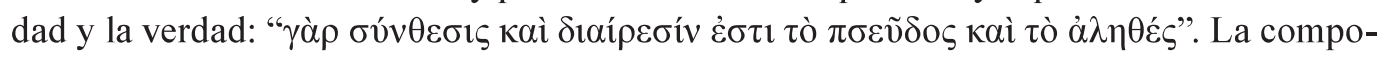
sición y separación resulta de un acto del entendimiento. "Así respectivamente puede ser verdadera o falsa una enunciación, como expresión de una composición o división, pero no puede serlo un nombre o un verbo tomado fuera del contexto de una enunciación"11. El

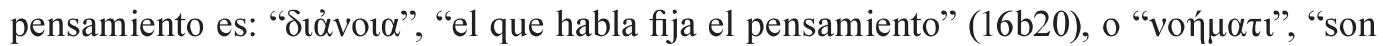
como los pensamientos que son sin combinación ni separación" (16a13) o de la misma raíz

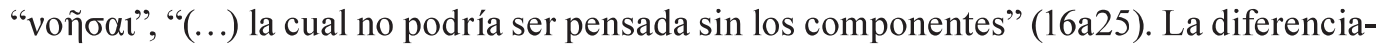
ción entre ambos términos no es muy explícita. En de Interpretación Aristóteles clarifica lo que estableció en las Categorías y a lo cual ya se refirió. La adición al nombre del verbo es esencial, sea el verbo ser u otros verbos. La bivalencia se desprende de la posibilidad

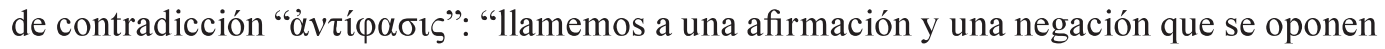
una contradicción" (16a32). En efecto la proposición declarativa puede ser afirmativa o negativa. Esta oposición es clave para entender la metodología de Aristóteles. El título que dio Teofrasto a uno de sus escritos donde se refiere al mismo tema fue: "Sobre la

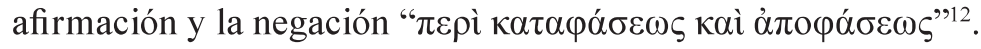

Hasta el capítulo 8 de de Interpretación, está vigente la lógica binaria que opone: la afirmación y la negación, la verdad y la falsedad. En la afirmación se puede dar la verdad así como la falsedad pero según lo que se da resulta el contrario en la negación correspondiente. O sea si una afirmación es falsa la negación del mismo asunto será verdadera. La oposición entre afirmación y negación tiene esta particularidad de expresarse en el verbo precedido o no por la negación: esto es la contradicción. Las proposiciones contrarias "Ėvavtía" que presentan oposiciones del sujeto o del accidente no presentan esta misma obligación de verdad o falsedad.

En el capítulo 9 de de Interpretación aparece un problema que va a romper el esquema

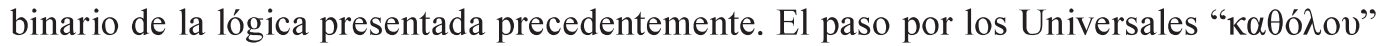
permite de confirmar la regla, ya que toda declaración acerca de un universal tiene que ser vigente o sea verdad o falsa independientemente del tiempo pasado, presente o futuro. En el caso de los particulares emerge un problema de peculiar importancia que no permite la aplicación del método por analogía: "Pero respecto de las singulares que están por ocurrir no es lo mismo" (18b34). Ya vimos que en las proposiciones declarativas el verbo consignifica el tiempo y se expresa en el presente, el pasado y el futuro siendo "inflexiones" (16b17). Aristóteles logra ajustar su método a todo hecho presente o pasado, pero para los hechos futuros se encuentra enfrentado al siguiente problema: o debe pretender a las de-

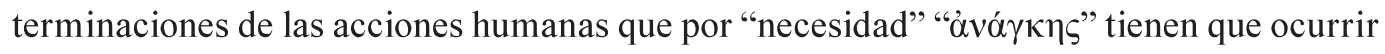

11 Skarica, M. (2003). "Enunciación aprehensiva y enunciación judicativa". Revista philosophica. Vol 26, p. 277.

12 Correia, M. (2003). La lógica de Aristóteles. Santiago: Ediciones Universitarias Católica de Chile, p. 51. 


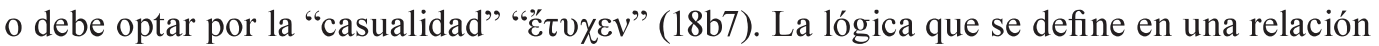
de causalidad escapa a la casualidad. "En el texto se declara que algún principio lógico no rige semejantemente para las aserciones singulares de tiempo futuro" 13 . Aristóteles claramente rechaza el determinismo por lo que concierne las acciones humanas. Luego la necesidad de que la afirmación o la negación sea verdadera o falsa ya no se confirma: "Thus he is denying that it holds of future singulars that each of a contradictory pair must be either truth or false"14. Aristóteles hace una relación entre la necesidad de que

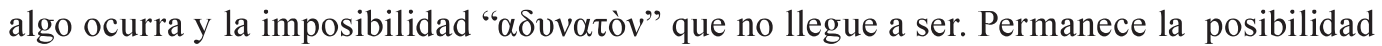

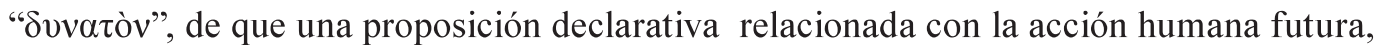
sea verdadera o falsa hasta que ocurra. Aristóteles introduce la noción de posibilidad que desarrolla más ampliamente como concepto de potencia en la Metafísica, libro IX, 1. La distinción entre actualidad / potentialidad está relacionada con el tiempo, la actualidad es

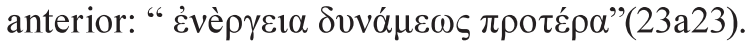

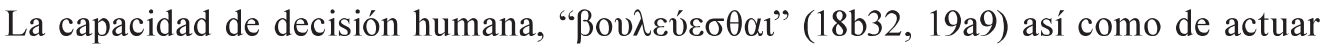

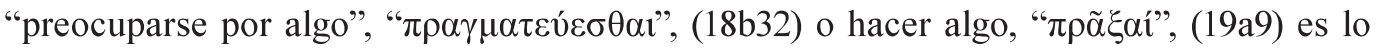
que va a subrayar los límites del método lógico. Es interesante observar que las relaciones a "las cosas" se invierten, las cosas anteriores al acto predicativo llegan a ser el objeto de la acción humana: las acciones van a influir en "los hechos". Luego la acción va a permitir en su tiempo de poder definir la verdad, la proposición futura, sin embargo, no se puede

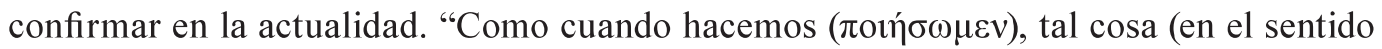
de producción) resulta tal cosa, y si no lo hacemos no resulta" (18b31). Aristóteles relaciona luego la verdad con el ser, existir de las cosas o de los hechos ( $\pi \rho \alpha ́ \gamma \mu \alpha \tau \alpha)$. Después de aludir al ejemplo de la posibilidad o no de ocurrir una batalla naval el día siguiente:

"de modo que, dado que las proposiciones son verdaderas de la manera como son los hechos así como hay sujetos que permiten que se den los contrarios y la casualidad, será necesario y del mismo modo, que se comporte también la contradicción” (19 a33).

Se vislumbra con parsimonia lo que va a ser explicitado en el tratado de la ética. Los

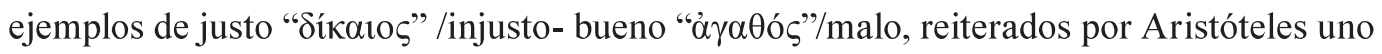
y otra vez en los últimos capítulos, pueden no ser fortuitos y anticipar lo que efectivamente será central en la ética: la orientación de la conducta humana hacia el bien y el practicar la justicia. Los ejemplos de justicia y bien, con sus contrarios, ya se encuentran como ejemplos en distintas ocasiones en las Categorías. Además en el capítulo XIV de de Interpretación Aristóteles entabla un análisis de cómo se introduce el juicio de la opinión o creencia " $\delta$ ó $\xi \alpha$ " refiriéndose a los ejemplos de justo y bueno y sus contrarios. Si bien es cierto que la autenticidad de este capítulo fue discutida, Boecio no dice nada al respecto y se apoya en los textos de Alejandro De Afrodisia ${ }^{15}$. Ackrill ${ }^{16}$ no pone en duda que Aristó-

13 Skarica, M. (1998). "Notas sobre de Interpretationes 9". En Aristóteles. Mendoza: Facultad de Filosofía y Letras, Universidad Nacional de Cuyo, p. 97.

14 Ackrill, J.L. (2002). Aristotle Categories and de interpretatione. New-York: Oxford University Press, p. 134. 
teles sea el autor pero sí que el capítulo XIV pertenezca a de Interpretación: muestra una incoherencia en el empleo de los términos de contrario y contradicción que sorprende allí ya que anteriormente Aristóteles los distinguió correctamente.

Los tratados de los Analíticos primeros y los Analíticos posteriores presentan el silogismo y su uso para la demostración en las ciencias. El silogismo " $\sigma 0 \lambda \lambda \sigma \gamma i \sigma \mu o ́ \varsigma "$ es un

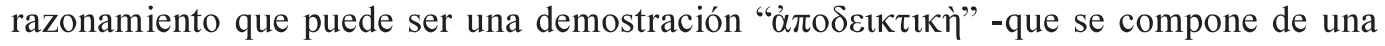

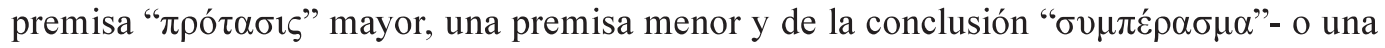

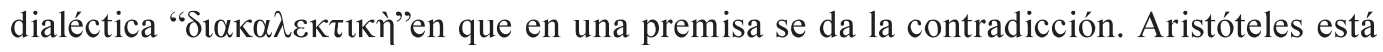
poniendo los cimientos de la metodología de la argumentación o de la dialéctica. La etimología de la palabra compuesta silogismo es pertinente: viene de " $\sigma v ́ v$ " "con" et $\lambda o ́ \gamma o \varsigma$ "palabra o discurso": sugiere la composición de frases de una con otra. El logos permanece en el tratado del Organon relacionado con la lingüística.

Ahora hacemos el salto en la Ética a Nicómaco para escudriñar allá las relaciones eventuales con la lógica. Aristóteles también escribió la Ética a Eudema y la Gran Moral, sin embargo me referiré a la Ética a Nicómaco.

\section{2) La Ética y el Bien en la Ética a Nicómaco. Función del deseo y de la virtud. La recta

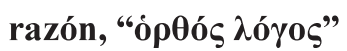

La Ética a Nicómaco ${ }^{17}$ es el primer gran tratado sobre la ética. La noción de Ética en la antigüedad se refiere a las relaciones que los hombres entretienen consigo mismo y con los

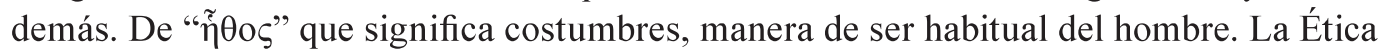
designa tanto un saber que se enraíza en la teoría como en la práctica y es en algún sentido un tratado político (EN 1094b5). Aparece una convergencia en una finalidad común: el

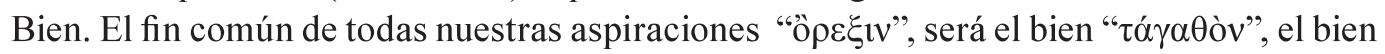

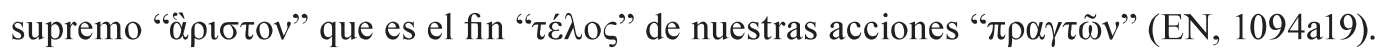
El conocimiento de este Bien resulta luego principal para la conducta humana. El bien supremo es la felicidad " $\varepsilon \hat{\jmath} \delta \alpha i ́ \mu \mu \omega v$ " pues se elige por ella misma. Aristóteles declara que si el bien es idéntico para el individuo y para el Estado "es evidente que es mucho más grande y más perfecto alcanzar y salvaguardar el de la ciudad" (EN 1094b7). El Bien no puede por otra parte ser definido universalmente ya que las opiniones respecto al bien son diferentes y hay distintos tipos de Bien.

Aristóteles critica a la teoría de las Ideas de Platón y opta por buscar la significación del bien a través de las categorías presentado en su primer tratado del Organon. "Se puede

15 Correia, M. La lógica de Aristóteles, op.cit., p 121.

16 Ackrill, J.L., Aristotle Categories and de interpretation, op.cit., p 153.

17 Versión en griego: Aristotle (1990). Nicomachean Ethics. (trad. in English H.Rackam, $11^{\circ}$ ed). London: Harvard University Press. Versión en español: Aristóteles. (1985). Ética Nicomáquea. Ética Eudemia. (trad. Julio Pallí Bonet). Madrid: Gredos. 
abrir una perspectiva interesante de aplicación de las categorías y de los relativos en una obra posterior de Aristóteles que es la Ética a Nicómaco"18.

"Además, puesto que la palabra "bien" se emplea en tantos sentidos como la palabra "ser" (pues se dice en la categoría de sustancia, como Dios y el intelecto "voṽ $\zeta$ "; en la

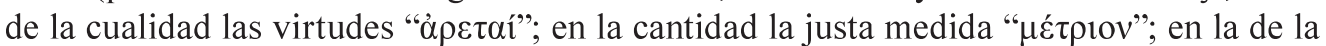

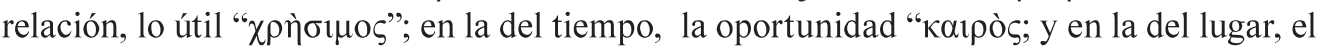
hábitat, y así sucesivamente) (EN 1096a24-28).

Aristóteles prosigue diciendo que no se puede definir el Bien en forma universal y única. "Ahora bien, conocer las cosas humanas en sí mismas contingentes de un modo universal, desde sus principios, significa renunciar de algún modo a su conocimiento perfecto"19.

Además el Bien se distingue para el hombre de distintas maneras: hay bienes exteriores, bienes del alma " $\pi \sigma v \chi \eta े v "$ y del cuerpo " $\sigma \tilde{\omega} \mu \alpha "$ (EN 1098b12). Los bienes del alma son principales. El alma dirige las acciones. Para precisar lo que es el Bien y llegar a los principios Aristóteles procede con metodología. Aristóteles nota que no sólo se puede detener en la conclusión y las premisas de la definición del bien supremo o felicidad " $\varepsilon v ̉ \delta \alpha i ́ \mu \mu \omega v$ " hay que considerar lo que se dice acerca de ello. "Pues con la verdad concuerdan todos los datos, pero con lo falso, pronto discrepan" (EN 1098b10-12).

La felicidad del hombre se obtiene por la virtud. La felicidad es lo propio del hombre. Aristóteles recuerda la necesidad de conocer el alma para poder dirigirla bien. Aristóteles retoma una presentación conocida del alma. Se divide en una parte que tiene la razón

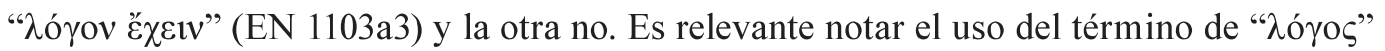

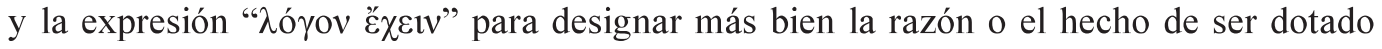
de razón. Aristóteles divide la parte irracional del alma también en dos partes: la parte

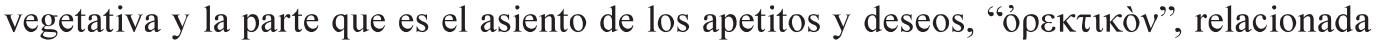
con la razón ya que le obedece "como se escucha a un padre" (EN1102b36). Lo particular del hombre adulto es de poder actuar en razón con virtud, no lo pueden, según él, ni los animales ni los niños.

Las virtudes son actividades del alma se diferencian de manera similar al alma

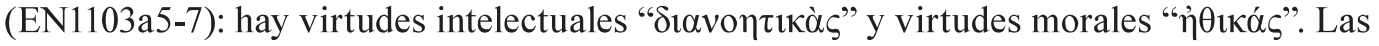

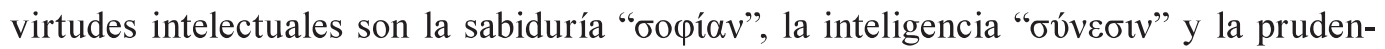

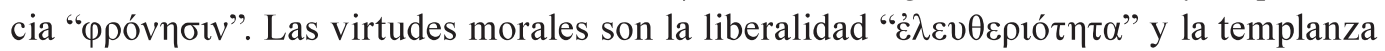

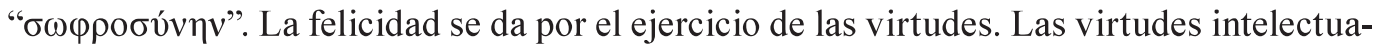

18 Furstenberg, C. (2014). "Los relativos en las categorías Aristóteles". EIKASIA. Vol 60. Recuperado de: http:// revistadefilosofia.com/numero60.htm : p. 213.

19 Yarza I. (2011) La racionalidad de la Ética de Aristóteles, Un estudio sobre Ética a Nicómaco I. Navarra: EUNSA, p. 21. 
les nacen y crecen gracias a la educación, las virtudes éticas proceden del hábito o costumbre (1103a15). Las experiencias así como el aprendizaje van a contribuir a mejorar las aptitudes a actuar con virtud. "La virtud es un modo de ser " $\varepsilon \xi \xi \varsigma$ " del hombre" (1106a14) modo de ser "por el cual el hombre se hace bueno, y realiza su función propia" (1106 a23). El modo de ser o hábito como " $\check{\xi} \xi ı$ " se encuentra ya en las Categorías (8b28) como cua-

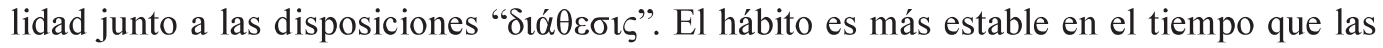
disposiciones. El hábito comprende a las virtudes y a los conocimientos que son estables en el alma y difíciles de ser modificados, mientras que las disposiciones varían y sufren cambios en caso de enfermedad, resfrió por ejemplo.

¿Qué es lo que en el hombre da la capacidad de ejercer la virtud?

"Commençons par ce qui paraît faire coupure dans l'Éthique à Nicomaque, à savoir la distinction entre vertus dites «éthiques» (Tricot) ou «morales» (Jolif) ( le prédicat éthique faisant référence à l'ethos, disposition pratique enracinée dans le désir), - et les vertus «intellectuelles» ou «dianoétiques», ainsi nommées parce qu'elles ont pour réfèrent 1 ' orthos logos, la règle juste, donc l'intellection à l'oeuvre dans l'action bonne» ${ }^{20}$.

En el libro II de la Ética a Nicómaco Aristóteles describe la acción de la recta razón o ó $\rho$ ós $\lambda o ́ \gamma o c:$ "Ahora bien, que hemos de actuar de acuerdo con la recta razón es comúnmente aceptado y lo damos por supuesto" (1103b32). Volverá a definirla y explicar sus relaciones con las virtudes. Mientras tanto, ya establece una relación entre la recta razón y el término medio ( $\mu \varepsilon \sigma o ́ \tau \eta \tau o \varsigma)$ entre dos extremos, el exceso ( $(\tau \varepsilon \rho \beta 0 \lambda \tilde{\eta} \varsigma)$ o el defecto

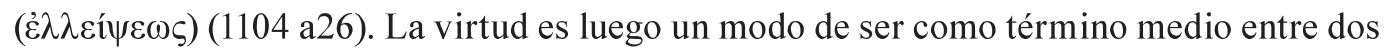
actitudes extremas.

En el libro VI de la Ética a Nicómaco la parte racional del hombre va a permitir determinar "un cierto límite de los términos medios que decimos se encuentran entre el exceso y el defecto y que existen en concordancia con la recta razón "ó $\theta$ ó $\varsigma \lambda o ́ \gamma o \zeta$ " " (EN 1138b23-25). Se define así una verdad que corresponde al justo medio que sin embargo no es suficientemente: conocerla no basta se debe comprender como funciona la recta razón.

"Lo que la afirmación y la negación son el en el juicio, el perseguimiento y la huida lo

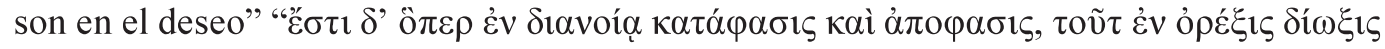

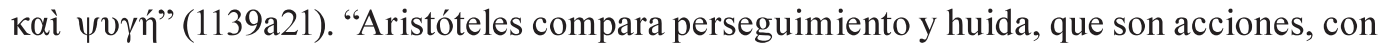
afirmaciones y negaciones" ${ }^{21}$ Aristóteles se refiere a la lógica binaria de la oración decla-

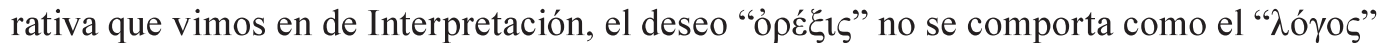
ya que no se logra una oposición tajante como la de la afirmación y negación sino que se tiende a un perseguimiento o a una huida.

20 Ricoeur, P. (1997). « A la gloire de la phronesis ». En La vérité pratique. Aristote, Ethique à Nicomaque, Livre VI . Textos compilados por Jean-Yves Château. Paris: Librairie Philosophique Joseph Vrin : 14.

21 Anscombe, E. (1998). "Verdad y razonamiento practico ». En Nuestros griegos y sus modernos. Estrategias contemporáneas de apropiación de la Antigüedad. Barbara CASSIN (comp.). Manantial: España, p. 286. 


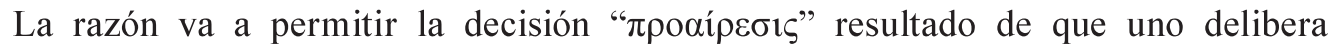

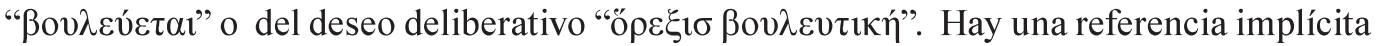
al capítulo 9 de De Interpretación: no se delibera de algo relacionado con el pasado sino de algo que concierne una acción futura y es posible que ocurra o no (EN 18b32, 19a9). Aristóteles introduce la deliberación como posibilidad propia del hombre cuyas acciones no son determinadas. Aristóteles confirma que la deliberación está relacionada con la acción que se realiza en el futuro, interviene allí la noción de finalidad " $\tau \varepsilon ́ \lambda o \varsigma$ " de Bien. "La délibération consiste à combiner des moyens efficaces en vue de fins réalisables. C'est donc que l'avenir nous est ouvert" ${ }^{\prime 22}$. Se puede decir que de la deliberación se obtiene en

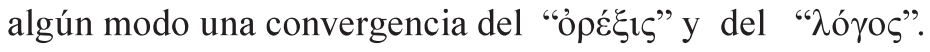

En de Interpretación no se encuentra la decisión " $\pi \rho \circ \alpha i ́ \rho \varepsilon \sigma ı "$, intermediaria entre la deliberación y la acción, en cambio está muy presente en la Ética a Nicómaco. La

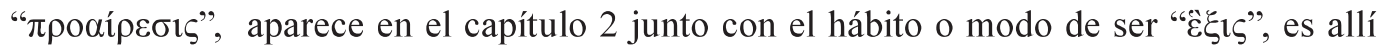
una disposición interior virtud moral. En el capítulo 6 es el resultado de la deliberación:

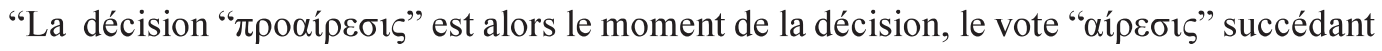
à la délibération, et qui n'est plus seulement la manifestation de l'intelligence délibérante, mais la volonté désirante, laquelle intervient pour mettre en branle la délibération, mais aussi pour y mettre fin" 23 .

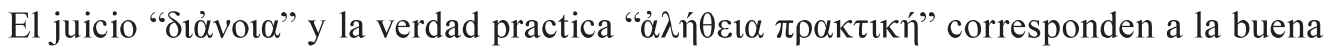
decisión "se trata de la verdad conforme al deseo bueno" (EN 1139a30). Aristóteles lo diferencia del juicio teórico en que se da la verdad y la falsedad como lo vimos en las oraciones declarativas en de Interpretación.

Aristóteles precisa que las capacidades intelectivas que permiten una razón recta y una buena argumentación no bastan para actuar bien. En efecto alguien puede obtener una argumentación y conclusión correcta pero por su vicio tener una acción pervertida. Por otra parte se puede obrar bien a pesar de tener una argumentación mala, sin embargo lo perfecto es la asociación de un juicio correcto con la virtud. Se requiere tener virtudes

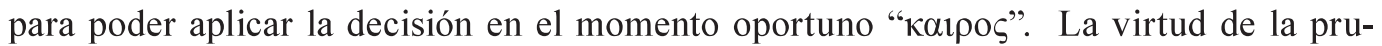
dencia " $\varphi \rho o ́ v \varepsilon \sigma 1 \zeta "$ " (EN 1144b2) es principal. La virtud de la prudencia es una virtud de deliberación que permite escoger los justos medios para alcanzar el fin. En el capítulo 7 de la Ética a Nicómaco (EN 1147a25-35). Aristóteles utiliza un método de argumentación el silogismo ya definido en el Organon, en el tratado de los Analíticos Primeros. Vigo ${ }^{24}$ deja claro el paralelismo entre el silogismo de moderación que resulta en una acción buena y el silogismo de incontinencia que resulta en una acción mala. En el silogismo de la moderación: la premisa mayor es el "deseo de abstenerse del dulce" la premisa menor: "esta torta

\footnotetext{
Aubenque, P. (1963). La prudence chez Aristote. Besançon : Presses universitaires de France, p. 112.

Aubenque, P. La prudence chez Aristote, op. cit., p. 121.

24 Vigo, A. (2006). Estudios Aristotélicos. Pamplona: EUNSA, p. 293.
} 
dulce" y la conclusión: no como. Luego el hombre actúa conforme a lo que resulta como bueno según el buen sentido " $\gamma v \omega ́ \mu \eta "$ " En el silogismo de la incontinencia "la premisa mayor: "deseo de probar dulce", la premisa menor "esta torta es dulce" y la conclusión "como", por la falta de la virtud de la templanza el hombre no se controla. La premisa mayor es desiderativa, la premisa menor volitiva y la conclusión activa.

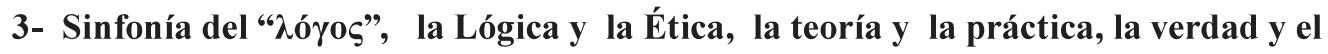
bien, entretejidos.

Intenté una lectura del tratado del Organon y en particular de Interpretación, estudiado en seminario, enriqueciéndola con un vínculo posible entrevisto con la obra de la Ética a Nicómaco. Procedí a la lectura de la Ética a Nicómaco buscando los rastros de la lógica. Esta experiencia me dio de entender mejor como procede Aristóteles, sus reflexiones se van afinando y, si cierto se separan en temas dominantes, estrellan constelaciones de referencias explicitas o implícitas hacia otros de sus temas o trabajos. Si el término de sinfonía me parece adecuado, es que refleja una cierta harmonía que se desprende de la evolución de su reflexión que tiene derecho a modularse según el punto de vista o el enriquecimiento de las experiencias.

El hilo conductor podría ser el " $\lambda o ́ \gamma o \varsigma$ ", cuya melodía varia sin perder una cierta coherencia o harmonía. En efecto se podría hacer un estudio mucho mas detallado de la evolución del sentido del " $\lambda o ́ \gamma o \varsigma$ " en la Obra de Aristóteles. Esto podría permitir, según mi punto de vista, una mejor comprensión de sus obras y ver que las huellas del logos permanecen presentes de una forma u otra. En efecto en de Interpretación el logos es mayormente en relación con el lenguaje, y en la Ética a Nicómaco, si permanecen algunas referencias donde el $\lambda$ ó $\gamma$ $\varsigma$ significa proposición, allí en general Aristóteles se refiere más

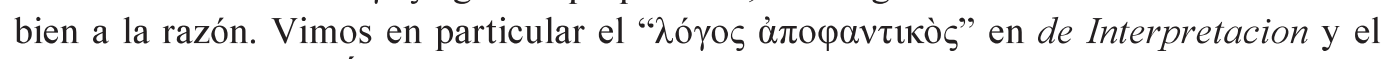

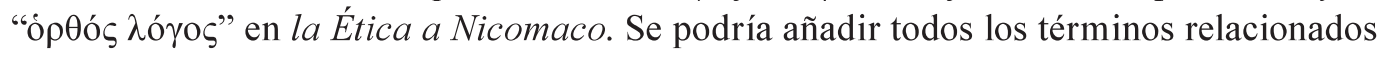
con el " $\lambda$ ó $\varsigma \varsigma$ " que Aristóteles utiliza: la dialéctica, el silogismo, la logística. El juicio que

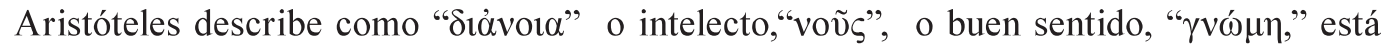
siempre en relación con el " $\lambda o ́ \gamma o \varsigma$ ", y revela lo que es propio del hombre según Aristóteles: la facultad de pensar. Hay que tener presente que en la antigüedad el " $\lambda$ ó $\gamma o \zeta$ ” era también representativo del orden cosmológico.

La Lógica, como instrumento metodológico, y la ética, como guía de la conducta humana, se complementan y permiten al hombre de alcanzar la felicidad en sociedad. La lógica interviene en la argumentación, en el conocimiento, la toma de decisión. La Ética, como orientación de la acción humana hacia el bien, da pautas para entender la importancia de la deliberación y de la decisión previa a la acción. La lógica y la ética así como la teoría y la práctica están vinculados. Los límites de la lógica presentados en el capítulo 9 de de Interpretación manifiestan el valor de la acción humana que no es determinada. 
Arendt en diversos escritos alude a la singularidad del pensamiento del hombre. El pensar otorga al hombre la facultad de dialogar consigo mismo y con los demás, le da la facultad del juico, de la decisión y motiva la acción. Frecuentemente se refiere a los escritos de Aristóteles y subraya la interacción entre la razón y el deseo así como la responsabilidad que incumbe al hombre por su capacidad de decidir.

"Ce qui se produit réellement, c'est que, puisque raison et désir sont en conflit, la décision en faveur de l'un ou de l'autre est affaire de "préférence", de choix et délibéré. C'est la raison qui intervient, par le nous attaché aux choses qui sont de tout temps en ne peuvent être autrement qu'elles sont, mas diainoia et phronesis, qui concernent les choses en notre pouvoir et se distingue en cela des désirs et effets de l'imagination, capables d'englober des choses que nous n'accompliront jamais, comme d'être des dieux ou immortels" 25 .

El bien como melodía de la Ética, y la verdad como pautas de la composición no se pueden separar según Aristóteles. En la lógica hay una verdad con su contradicción posible, la falsedad, existe también la conclusión verdadera del silogismo. En la ética hay una verdad práctica que corresponde a la acción buena resultado de una recta deliberación, existe también el silogismo práctico bueno. La finalidad hacia el bien tiende al futuro, la verdad tiende más bien a su actualización en el presente.

Non obstante se puede decir que hay anterioridad de la lógica sobre la Ética según la lectura de de Interpretación. Non obstante la lectura de la Ética a Nicómaco da la impresión inversa, la lógica viene en ayuda como metodología útil de la razón para acertar en las decisiones y actuar bien. Así mismo en la Ética a Eudemia (EE 1216b27-1217a19) describe el papel de la argumentación para descubrir la verdad y su importancia en las relaciones humanas.

Conviene observar que la meta del hombre es la felicidad y que la Lógica y la Ética se aplican en la vida personal tanto como en la vida en sociedad o en la Política. Aristóteles lo recuerda tanto al principio como al final de la Ética a Nicómaco. Entonces la educación, según él, debe incluir la lógica y la ética. Así se permite de adquirir hábitos apropiados así como comportamientos buenos.

Se encuentra en la obra de Stein un análisis de la relación entre la verdad y el bien que corrobora la posición de Aristóteles aunque se define en sus escritos una resonancia teológica:

"El sentido de verdadero y bueno permanece, sin embargo, diferente igual que el conocimiento y la tendencia son distintos y, sin embargo, están en el mismo tiempo

Arendt, H. (2007). La vie de l'esprit. Paris : Presses Universitaires de France, p. 350. 
condicionados el uno por el otro" (...) "Cuando el médico reconoce que el enfermo podría curarse por un descanso en la montaña, sabe que el descanso es un bien para el enfermo. Su entendimiento (y, cuando ha transmitido este mismo conocimiento al enfermo, el entendimiento también de este último) ha recibido un enriquecimiento, aun si el descanso no se realiza. Pero el bien que existe para los dos fines de la tendencia, a saber de la salud, no puede ser causado más que por el descanso real"26.

Como llave de Sol o primicia de experiencia humana espiritual le invito a una conside-

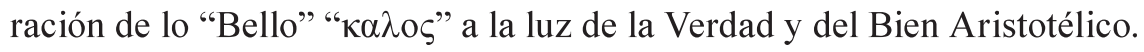

"La belleza tiene un rasgo común con la verdad y la bondad: pone al ente en relación con un ente determinado, y específicamente- de nuevo, al igual que la verdad- en relación con el espíritu: en efecto, la belleza es lo que hace al ente susceptible de despertar placer, pero el placer es un acto espiritual" ${ }^{27}$.

\section{Conclusión}

La perspicacia de Aristóteles es notoria y su aptitud en crear, sin tregua, relaciones entre sus distintas temáticas de reflexión, notable. La lectura de de Interpretación y de la Ética a Nicómaco se enriquecen mutuamente y permiten comprender la dinámica reflexiva de Aristóteles que no tiende a una separación temática sino más bien a un entretejido sutil. Entre la lógica y la Ética, la teoría y la práctica, la verdad y el bien, no hay fronteras nítidas, sino más bien un entramado. En la excelencia del actuar humano se puede decir que la verdad y el bien se asimilan de alguna forma conservando a su vez su sentido propio.

La lectura del capítulo 9 de de Interpretación de Aristóteles ha sido el objeto de comentarios profusos y variados tanto en filosofía como en teología con múltiples debates sobre el libre albedrio (comentarios de Ammonio, Boecio, Sto Tomas de Aquino, Lukasiewicz, Russel, Hume, etc). El capítulo 6 de la Ética a Nicómaco suscitó muchos trabajos sobre la libre decisión, la deliberación y la verdad práctica. La relación entre ambos capítulos es patente y se puede, como lo vimos en este trabajo, entrever lazos explícitos e implícitos en el conjunto de las obras de la Lógica y de la Ética.

Hay dos orientaciones posibles: una que alimentaria una reflexión sobre el pensamiento y juicio personal, donde la dialéctica es interior y otra que concierne la dialéctica en los discursos humanos y en la política. "Sans conscience, au sens de conscience de soi-même, la pensée ne serait pas possible (...) Pour Socrate, la dualité du deux en un signifiait tout

26 Stein, E. (1996). Ser finito y ser eterno. (trad. Alberto Pérez Monroy). México: Fundo de Cultura económica: pp. 329-330.

27 Stern, E. (1996). Ser finito y ser eterno. (trad. Alberto Pérez Monroy). México: Fundo de Cultura económica: pp. 337. 
simplement que si l'on veut penser, il faut s'arranger pour que les deux interlocuteurs soient en bonne formes et soient amis l'un de l'autre" 28 . Aristóteles en el capítulo 9 de la Ética a Nicómaco solicita una reflexión sobre la amistad que permite la concordancia entre los seres humanos, alude a la amistad entre prójimos pero también a la amistad civil. Si el trato entre los seres humanos se regula por el lenguaje también lo es por el comportamiento. La búsqueda de la verdad del conocimiento permite una adecuación a los misterios de la realidad la más acertada posible. Los deseos humanos atisbados por las sensaciones y percepciones necesitan ser analizados por la razón para optar por la acción buena. Además, para que la acción buena se cumple se necesita la virtud. Las virtudes como disposiciones humanas se adquieren y se fomentan mediante la educación.

Hay un interés actual de la lógica y ética Aristotélica en las ciencias de la salud. La lógica es el método propio de las ciencias, sin embargo tiene sus límites en el campo de las realidades humanas y en particular en lo que atañe a las decisiones futuras. Hay estudios interesantes donde neurociencias y filosofía intentan una complementariedad. En efecto, a pesar de los múltiples progresos de los conocimientos del funcionamiento del sistema cerebral y neuronales, la lógica científica no logra a determinar el ejercicio del libre albedrio $^{29}$. La virtud de prudencia a su vez es particularmente útil en las tomas de decisiones medicales, "L'action résultant de la prudence n'est pas non plus une simple velléité mais un acte engagé" ${ }^{30}$. La acción apela a la responsabilidad. Las decisiones medicales además implican a tercero involucrado: el paciente. Si la ciencia ofrece pronósticos detallados y a veces programados según una evolución "lógica" resulta que las realidades humanas deciden o resultan otras. La adaptación de la decisión médica es difícil y compleja ya que tiene que tomar en consideración la persona en su integridad física, psíquica, espiritual, cultural. También los datos de las ciencias son importantes ya que permiten prevenir o anticipar pero la forma de vivirlo permanece personal. El análisis tiene que tomar en cuenta el conjunto de los datos clínicos, personales, familiares, sociales. "Es, por tanto, la virtud un modo de ser selectivo, siendo un término medio relativo a nosotros, determinado por la razón y por aquello por lo que decidiría el hombre prudente" (EN 1106b36-38). Tanto en de Interpretación como en la Ética a Nicómaco Aristóteles utiliza bastantes ejemplos relativos a la medicina. Se merece proseguir indagando en los intereses de trabajos en común entre ciencias, neurociencias y filosofía.*

28 Arendt, H. (2007). Op.cit.

29 Giménez-Amaya, J.- Murillo, J. (2009/1) "Neurociencias y libertad, una aproximación interdisciplinar". SCRIPTA THEOLOGICA 41, pp. 13-46.

30 Furstenberg, C. (2011) L'autonomie s'amenuise, la vulnérabilité affleure, la personne demeure. PARIS: Beaurepaire, pp. 187-190.

* Artículo recibido: $1^{\circ}$ de mayo de 2015. Aceptado: 27 de julio de 2015. 
De la Lógica a la Ética. La sinfonía del Lógos en Aristóteles

\section{Bibliografía}

Ackrill, J. L. (2002). Aristotle Categories and de interpretatione. New-York: Oxford University Press.

Anscombe, E. (1994). «Verdad y razonamiento practico ». En Nuestros griegos y sus modernos. Estrategias contemporáneas de apropiación de la Antigüedad. Barbara CASSIN (comp.). Manantial: España, 281-290.

Arendt, H. (2007). La vie de l'esprit. Paris : Presses Universitaires de France.

Aristoteles. (1985). Ética Nicomaquea. Ética Eudemia. (trad. Julio Pallí Bonet). Madrid: Gredos

Aristoteles. (1990). Nicomachean Ethics. (trad. in English H.Rackam,11ed). London: Harvard University Press.

(1983). The cathegories on interpretation Prior analytics. ( $\left.7^{\circ} \mathrm{ed}\right) . \mathrm{By}$ H.P.Cooke \& H.Tredennick. London : Harvard University Press.

Aubenque, P. (1963). La prudence chez Aristote. Besançon: Presses universitaires de France.

(2009). « Sens et unité du traité Aristotélicien de l'Interprétation ». En Interpréter de Interpretatione de Suzanne Husson. Paris: Vrin, 37-50.

Correia, M. (2003). La lógica de Aristóteles. Santiago: Ediciones Universitarias Católica de Chile.

FurstenberG, C. (2014). "Los relativos en las categorías Aristóteles". EIKASIA. Vol 60 : 199-220 Recuperado de: http://revistadefilosofia.com/numero60.htm: 201-222.

, (2011). L'autonomie s'amenuise, la vulnérabilité affleure, la personne demeure. PARIS: Beaurepaire

GimÉnEZ-AmAYA, J. y Murillo, J. (2009/1) "Neurociencias y libertad, una aproximación interdisciplinar". SCRIPTA THEOLOGICA $41: 13-46$

HAdot, P. (1980) « Sur divers sens du mot pragmata dans la tradition philosophique grecque ». En concepts et catégories dans la pensée Antique ». Por Pierre Aubenque. Paris: Vrin, 312-316. 
Cécile Furstenberg

GimÉnez-Amaya, J. y Murillo, J. (2009/1) "Neurociencias y libertad, una aproximación interdisciplinar". SCRIPTA THEOLOGICA 41 13-46.

Joly, A. (2008). « Notes de lectures: de quelques malentendus sur la définition du verbe ». ERIS : 139-149.

Mittelmann, J. (2009). Categorías sobre la interpretación. Buenos Aires: Colección Griegos y latinos, Losada.

Moreau, J. (1980). «Aristote et la vérité antéprédicative ». En Aristote et les problèmes de méthodes. Paris : Vrin, 21-33.

Ricoeur, P. (1997). «A la gloire de la phronesis ». En La vérité pratique. Aristote, Ethique à Nicomaque, Livre VI . Textos compilados por Jean-Yves Château. Paris: Librairie Philosophique Joseph Vrin, 13-22.

SKarica, M. (2003). "Enunciación aprehensiva y enunciación judicativa". Revista philosophica. Vol 26.

(1998). "Notas sobre de Interpretationes 9". En Aristóteles. (Actas de las Primeras Jornadas Aristotélicas Argentinas, Mendoza, 1996). Mendoza, Universidad Nacional de Cuyo: H. J. Padron, 97-102.

Toulon-Var : Equipe d'accueil Babel 2649. Association Modèles linguistiques. Recuperado de : http://ml.revues.org/269

VIGO, A. (2006). Estudios Aristotélicos. Pamplona: EUNSA.

Weidemann, H. (2014). Aristóteles de Interpretatione. Berlin: De Gruyter.

YARZA, I. (2011). La racionalidad de la Ética de Aristóteles. Un estudio sobre Ética a Nicómaco. Navarra: EUNSA. 